\title{
NEED FOR RELIABLE SENSOR CALIBRATION FROM THE PERSPECTIVE OF A NATIONAL MAPPING AGENCY
}

\author{
S. Baltrusch ${ }^{\text {a }}$ \\ a LAiV M-V, NMCA M-V, Department for Photogrammetry, 19059 Schwerin, Germany - sven.baltrusch@laiv-mv.de
}

\section{EuroCOW 2016}

KEY WORDS: Calibration, Validation, Geometry, Radiometry, standardisation, Basic Geodata, NDVI

\begin{abstract}
:
The sensor calibration is one of the basic elements for getting effective and efficient production workflows out of airborne photogrammetry. Digital images and their orientations (interior and exterior) are the key to get the resulting products into the workflow of a national mapping agency (NMCA). Not only the geometric calibration is required meanwhile the radiometric calibration as well is used, for example in raster-based classification processes. In the paper the requirements are shown and examples are presented. At least open aspects and the outstanding debts are given.
\end{abstract}

\section{INTRODUCTION}

\subsection{Current position}

The NMCAs of the Federal Republic of Germany contract out aerial surveys since many years. In the past the most and nearly only aim was to update the information in the landscape model (Basis-DLM) as well as in the series of topographic maps.
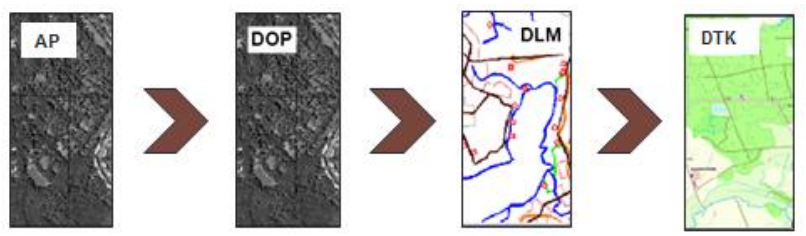

Figure 1. Traditional workflow for aerial survey results in NMCAs

The aerial photos still deliver fundamental information for the Authorative Topographic-Cartographic Information System ATKIS (Figure 2).

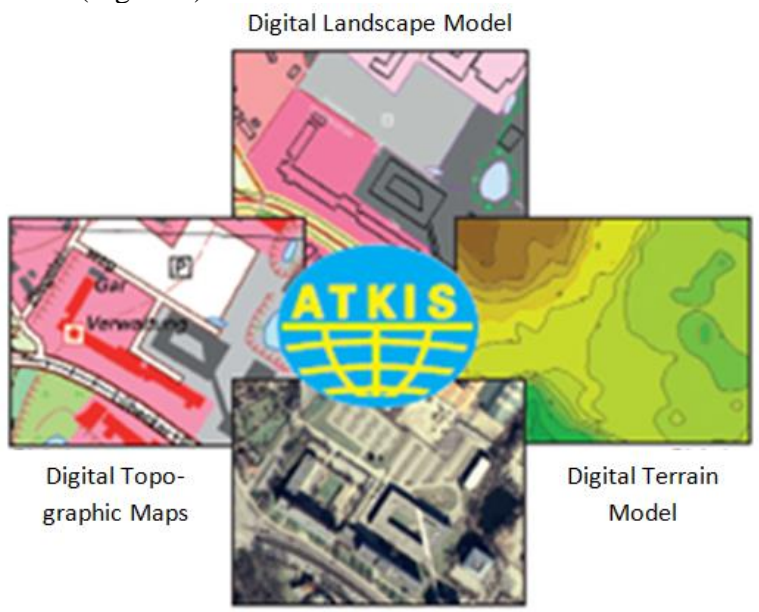

Digital Orthophotos

Figure 2. Authorative Topographic-Cartographic Information System ATKIS
With the change into digital photogrammetry workflows and the new basic approaches, deduced from the computer vision technologies, the aerial photos gained from aerial surveys have an increasing importance for a variety of applications. The quality demands have increased and the currency cycles were shortened parallel. These new use cases strengthen the need for reliable sensor calibration. Not only the geometric calibration is required meanwhile the radiometric calibration as well is used, for example in raster-based classification processes.

The NMCAs have to reach these requirements with respect to permanently shrinking number of staff, which requires automatic workflows, control steps and monitoring strategies. Only samples are possible. Sustained quality controls are inefficient and are extremely time-consuming. Figure 3 shows the controlpoint distribution for NMCA aerial survey projects (JRC, 2008).

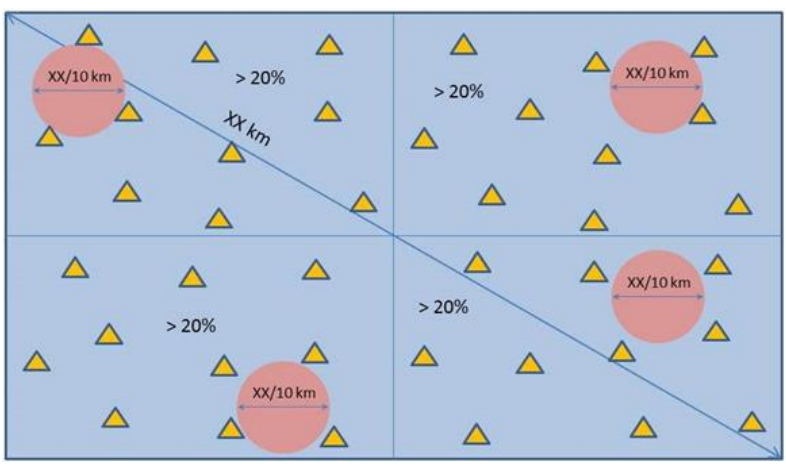

Figure 3: Controlpoint distribution for NMCA aerial survey projects

Other official agencies depend on a continuous delivery of qualified and contemporary datasets for their own purpose, commonly indentured by European or national laws. 


\subsection{Aerial Survey parameters of a NMCA}

The German NMCAs have to tender their aerial survey projects with respect to the national and the European public procurement law. The Working Committee of the Surveying Authorities of the States of the Federal Republic Germany $(\mathrm{AdV})$ published a guideline for tendering a digital aerial survey as a basis for the production of ATKIS-DOP and stereoscopic analysis (AdV, 2014).

The requirements reflected to the camera-system are described with the following facts:

- $\quad$ Large format digital camera systems

- Simultaneously recording of the panchromatic, the red, green and the blue channel as well as the near infrared with separated optics

- PAN-sharpening-ratio max. 1:4

- Illumination with a leaf shutter system

- Motion blurring has to be minimized with FMC/TDI and gyro techniques

- Orientation accuracy of the direct georeferencing shall be better than $0,5 \mathrm{~m}$ spatial variance

The following technical parameters complete the definition of an aerial project:

\begin{tabular}{|l|c|}
\hline Parameter & Value \\
\hline Geometric Resolution & GSD $10-20 \mathrm{~cm}$ \\
Spectral Resolution & PAN + RGBI \\
Radiometric Resolution & $8 / 16 \mathrm{bit}$ \\
Forward Overlap & $70-80 \%$ \\
Side Overlap & $30-50 \%$ \\
Flight cycle & $2-3$ years \\
Assignment period & Spring / Summer \\
\hline
\end{tabular}

Table 1. Aerial survey parameters of a NMCA

\subsection{Calibration requirements}

The camerasystem has to be calibrated geometrically and radiometrically. The requirements are based on the remarks given in the DIN 18740-4. The calibration has to be proven by a certificate of the constructor. The validity of the geometric calibration for the time of an aerial survey has to be attested by a validation flight (not older than one year) or a renewed complete calibration (not older than two years).

\subsection{Orientation requirements}

For the image orientation the direct georeferencing (GNSS, INS) has to be used. The correction parameters of the German SAPOS-Service (DGNSS-permanent-services of the NMCAs) have to be considered. The orientation accuracy resulting from the direct georeferencing has to be better than $0,5 \mathrm{~m}$ spatial variance. The evidence has to be provided by the contractor at a spatial distributed selection of ground control points appropriated by the NMCAs. If the required accuracy cannot be provided the contractor has the duty to perform an aerotriangulation.

\section{AUTHORITIVE PRODUCTS}

\subsection{The relevance of aerial photos}

The basic product in the photogrammetric workflow in German NMCAs is the dataset of aerial photos. These photos have a fundamental relevance, because of

- no loss of information,

- no loss of projection,

- no loss of interpretation,

- no loss of dimensionality.

Thanks to the increasing accuracy of the direct georeferencing and the developments on the hardware consumer market as well as to the sinking prices for stereo visualisation techniques the aerial photos celebrated a renaissance.

\subsection{Orientated aerial photos}

Aerial photos with all necessary parameters for a stereoscopic evaluation (especially interior and exterior orientation) are called orientated aerial photos. The definitions, quality requirements and metadata definitions are based on the AdVStandard for digital aerial photos (AdV, 2015).

The orientated aerial photos are divided in classes as a function of their orientation accuracy $(1 \sigma)$ :

LB1: image center (X, Y) approximated

LB2: 3-fold $\mathrm{GSD}^{1}$ (resulting from direct georeferencing)

LB3: 2-fold GSD (resulting from aerotriangulation)

LB4: 1-fold GSD (resulting from aerotriangulation)

\begin{tabular}{|c|c|c|}
\hline $\begin{array}{c}\text { Accuracy- } \\
\text { classes }\end{array}$ & GSD10 & GSD20 \\
\hline LB1 & $1,00 \mathrm{~m}$ & $1,00 \mathrm{~m}$ \\
\hline LB2 & $0,30 \mathrm{~m}$ & $0,60 \mathrm{~m}$ \\
\hline LB3 & $0,20 \mathrm{~m}$ & $0,40 \mathrm{~m}$ \\
\hline LB4 & $0,10 \mathrm{~m}$ & $0,20 \mathrm{~m}$ \\
\hline
\end{tabular}

Figure 4: Productclassification, defined by orientation accuracy

\subsection{Production chain}

The information of orientated aerial photos lead into a production chain with a series of following products like (Figure 5):

- Preliminary Orthophotos without interactive correction steps in geometry and radiometry (DOP-V)

- Orthophotos with interactive corrections (ATKISDOP)

- Rasterbased classification (Imageb. Classification)

- Dense Image Matching - Surface models (BDOM)

- Digital Terrain Models (ATKIS-DGM)

- Digital Surface Models (AdV-DOM)

- 3D-Building-Models (AdV-3DGbm)

- TrueOrthophotos (True-DOP)

- $\quad$ Timedifferenced Surface Models (tDOM)

${ }^{1}$ GSD $=$ Ground Sample Distance 


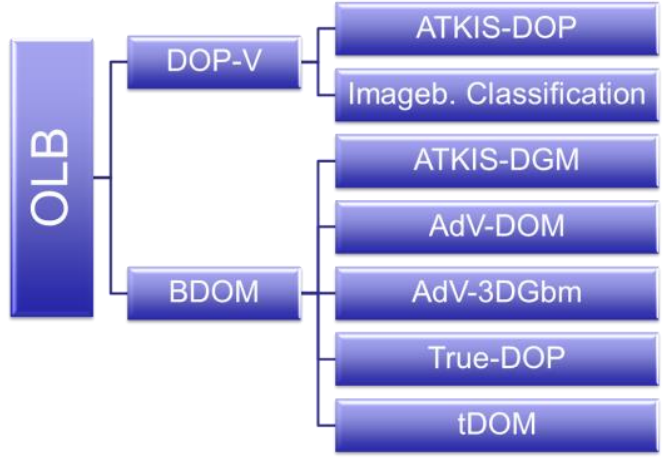

Figure 5: Product chain based on orientated aerial photos

These so called official basic geodatasets (Amtliche Geobasisdaten) are used in a lot of technical applications at other administrative bodies. Certified with the label "official product" they stand for neutrality on interests, reliability, unity in quantity and quality. These datasets have to be gapless and nationwide.

This induces the need for reliable sensor calibration from the perspective of a national mapping agency.

\subsection{Additional requirements reasoned by European wide access to camera systems}

The increasing accuracy requirements and the combined variety in the product chain lead to additional conditions of the camera system. The access and availability of the systems has to be made sure in the assignment periods.

Usually there are only a few days with weather conditions for aerial surveys so that the system has to guarantee stable calibration parameters for a complete assignment period. No additional camera calibrations are possible in "flight seasons".

The stability has to be made sure for a mission of several hours, only with stop-over for refueling.

The system parameters also have to be stable for varieties of weather conditions as well as in various flying heights caused by different target resolutions or restrictions of the aviation safety, for example at international airports. These parameters also depend on different conditions in different regions, for example depending on the changes of the relief.

\section{EVERYDAY PROBLEMS}

\subsection{Default of standardised calibrations}

In comparison to the analogue camera generation there is no standardisation of camera calibration anymore. The camera owner charges the camera constructor with the realisation of the camera calibration. Some owners do a calibration every two years, some owners perform a validation flight once a year.

The content of the calibration- or validation certificate varies between the camera constructors. Supplementary it has to be considered that the certificate is issued by the constructor and not an independent agency.

Especially the information resulting from a validation flight is commonly very sparse and not meaningful. The validation of the radiometric characteristics is completely missing. The procedure of the flight is not predefined. There are no specifications for standardised flying heights, scope and so on.

In the task force "ATKIS-DOP" of the AdV a requirement specification for the performance of a validation flight is developed in association with scientists of TU Dresden (AdV, Prof. Maas, 2015).

- After every massive reconstruction at the camera system a new calibration has to be performed.

- The documentation of a validation check bases on the DIN 18740-4. The documentation has to give a replicable evidence, that the position- and height accuracy of the camera system worsens not more than $25 \%$ in comparison to the reference survey (burn-in flight).

- Precondition is a „,burn-in flight“ just after the last camera calibration. These results are used as a reference for the qualitive assessment of the validation flight. A comparison is factual, if both flights - burn-in flight and validation flight - are similar according to image scale, block configuration, use of control points and loading of all observation groups.

- Besides the full documentation of the bundel block adjustment, the graphical presentation of the flight structure, the control- and checkpointdistribution the following information have to be delivered:

$\begin{array}{cl}\circ & \text { Flying height or GSD (max. deviation 20\%) } \\ \circ & \text { Forward overlap (max. deviation 10\%) } \\ \circ & \text { Side overlap (max. 5\%) } \\ \circ & \text { Number of photos (max. 50\%) } \\ \circ & \text { Number of flight strips (min. } 3 \text { strips) } \\ \circ & \text { Number of cross strips (min. 1 strip) } \\ \circ & \text { Number of control points (max. 8 points) } \\ \circ & \text { Number of check points (min. 15 points) } \\ \circ & \text { Identical configuration for GNSS/INS }\end{array}$

The deviation always refers to the comparison between the burn-in flight and the validation flight.

The processes of the aerotriangulation including the strategies (loadings, camera calibration yes/no, boresight alignement yes/no) have to be similar.

Finally the results from the statistics have to be compared. Both the internal values of the bundle block adjustment (for example: weight unit error) and the values of independent external reference measurements are expected to be similar with maximum deviations of $25 \%$.

- For the assessment of the validation results and the compliance of the calibration values the documents belonging to the camera calibration as well as the results of the burn-in flight are needed.

These additional requirements are mandatory for the processing of validation flights for aerial surveys commissioned by a German NMCA.

\subsection{Radiometric calibration}

For the radiometric calibration a labour calibration is needed. In a camera validation these process is not included. Nevertheless the analysis and the use of the multispectral information growes constantly.

With respect to the multispectral information in a digital aerial survey with a large format camera system, the NMCAs try to adapt remote sensing algorithms used for satellite images on the analysis of aerial photos. Combined with other basic geodata information - mostly obtained from the same photo sources - it is possible to build up a multidimensional feature space for rasterbased classification applications. 


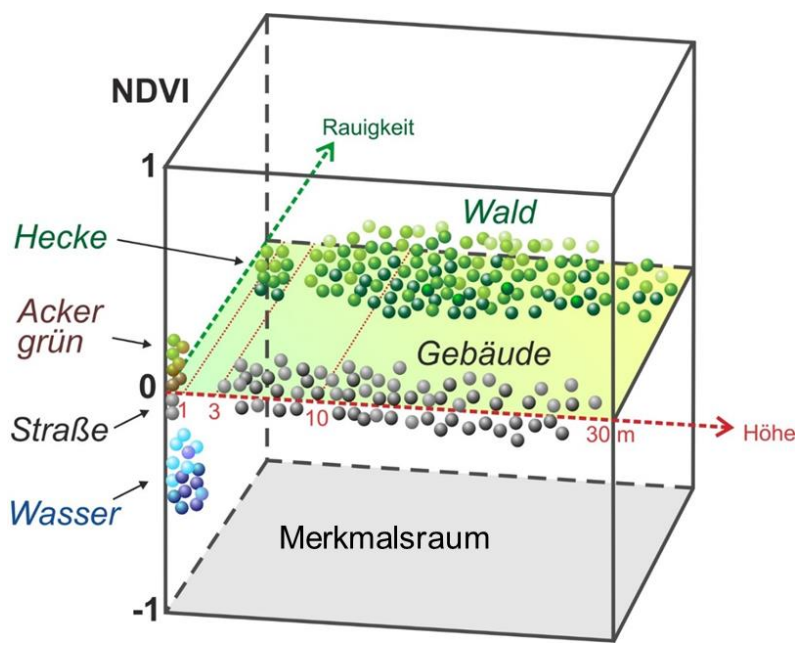

Figure 4. Multidimensional feature space (Rzeznik, 2015)

Current researches try to use these methods for updating the information in the landscape model, especially the updating of the effective landuse. This process demands a lot of man-power in interactive work.

For effective results the radiometric characteristic of the camera system has to be stable and reliable.

\subsection{Requirements to post-processing-steps in camera software}

Usually the task for the aerial survey contractors is to deliver aerial photos with a homogenous radiometric presentation for orthophoto productions. Therefore post-processing steps generate survey photographs from raw data. These production steps ignore the ratio between the red and the near infrared channel, which is a basic function in imagebased classification. This neglection leads to wrong assumptions based on the NDVI. In an aerial survey, flown at one day no constant NDVI values for the same objects are guaranteed.

\subsection{Camera calibration in aerotriangulation steps}

The NMCAs try to avoid camera calibration steps during an aerotriangulation. These calibration steps can be so powerful, that the final result, represented at a small number of control points, looks better than the projectwide quality really is.

Experiences of past projects show results, where the effects are not reasonable. A shift in the focal length or in the z-values lead to better results. But this is not justifiable.

The self-calibration is the last step to get better results. Normally geometric calibrated cameras are expected. If the camera self-calibration works effective enough there won't be the demand for labour calibrations anymore.

\subsection{No standardised exchange formats for camera parameters}

Regardless on the discussions of the advantage and disadvantage of the common parametersets resulting from camera calibration in the aerotriangulation process there is no standardised exchange format for these parameters. This implicates that the accuracy improvements normally only can be used in the software solution similar to the aerotriangulation software. A change in the processing software with a reimport of the adjusted images is not solved at the moment.
But in the era of the renaissance of the orientated aerial photos non photogrammetric specialised software solutions want to use this information as well.

Additional analysis according to the stability of the radiometric sensor characteristic have to be implemented in the validation process.

\section{CONCLUSIONS / OPEN TOPICS}

The developments and requirements to digital photogrammetric products increased the last years very strong. This leads to high requirements according to the input data. The fundamental datasets are the orientated aerial photos with their geometric and radiometric information. The datasets have to be reliable calibrated so that the NMCAs can start their production workflows without attempts of camera improvements.

The NMCAs are looking forward to:

- Standardisation in camera calibration, preferably done by an independent agency,

- Implementing radiometric calibration into validation processes,

- $\quad$ Regard on the ratio (red / near infrared) in post-processing steps in camera software

- $\quad$ Standardised exchange-format for orientated aerial photos

\section{REFERENCES}

AdV, 2014. Guideline for tendering a digital aerial survey as a basis for the production of ATKIS-DOP and stereoscopic analysis (adv-online.de).

AdV, 2015. AdV-Standard for digital aerial photos (advonline.de).

DIN18740-4:2007-09: Photogrammetric products - Part 4: Requirements for digital aerial cameras and digital aerial photographs.

JRC, 2008: Guidelines for Best Practise and Quality Checking of Ortho Imagery - Issue 3.0

Rzeznik, 2015: Change Detection from official remote sensing datasets (unpublished) 